Original Article

\title{
Application of Thoracoscopic Hybrid Surgery in the Treatment of Stage III Tuberculous Empyema
}

\author{
Zhao Liu, PhD, ${ }^{1}$ Sizhe Cao, MM, ${ }^{2}$ Changsheng Zhu, MM, ${ }^{1}$ Lin Wei, MM, ${ }^{1}$ \\ Huijun Zhang, MM, ${ }^{1}$ and Qian Li, $\mathrm{MM}^{1}$
}

\begin{abstract}
Background: To investigate the efficacy and value of thoracoscopic hybrid surgery in the treatment of stage III chronic tuberculous empyema (CTE).

Methods: 48 patients diagnosed as CTE with pleural thickening and encysted abscess cavity from were treated by hybrid operation (HO). Small incision operation was first used for resection of thickening pleural fibreboard and decortication of parietal pleura. Then, thoracoscopy was guided into chest to decorticate the visceral pleurali. Additional 25 patients with open operation of pleurectomy were set as control.

Results: The average operation time of $\mathrm{HO}$ group was $70 \pm 22 \mathrm{~min}$ compared to $130 \pm 32 \mathrm{~min}$ of control. The amount of bleeding, hospitalization time and chest tube drainage of $\mathrm{HO}$ group ( $200 \pm 55 \mathrm{ml}, 18 \pm 1.2$ days, $3.5 \pm 1.5$ days) were significantly decreased compared to control $(400 \pm 45 \mathrm{ml}, 28 \pm 4.5$ days, $6.5 \pm 2.5$ days). Post operation complications occurred in 5 $(\mathbf{1 0 . 4 2 \% )}$ ) and $3(12 \%)$ cases for HO group and control, respectively.

Conclusions: In stage III CTE, the small incision assisted thoracoscopic hybrid surgery help to remove thickening parietal pleura, promote the application of thoracoscopy, which has obvious advantages compared to traditional surgery.
\end{abstract}

Keywords: thoracoscopy, tuberculosis, surgery incisions

\section{Introduction}

Tuberculous empyema is a common disease of tuberculosis, due to mycobacterium tuberculosis and its secretion invade into the chest which caused specific suppurative inflammation. Mycobacterium tuberculosis caused thorax infection by lymphatic or blood circulation, pulmonary lesions direct invasion, or empyema formed by lymphatic tuberculosisor or bone tuberculosis abscess rupture into the pleural. Research ${ }^{1)}$ showed that nearly $90 \%$ tuberculosis

${ }^{1}$ Department of Thoracic Surgery, Xi'an Chest Hospital, Xian, China

${ }^{2}$ Department of Medicine, Xi'an Chest Hospital, Xian, China

Received: May 20, 2015; Accepted: July 18, 2015

Corresponding author: Zhao Liu, PhD. Department of Thoracic Surgery, Xi' an Chest Hospital, No. 127, Changan South Road, Xi'an 710061, China

Email: zhao_liu_au@163.com

(C)2015 The Editorial Committee of Annals of Thoracic and Cardiovascular Surgery. All rights reserved. empyema has a history of tuberculous pleurisy, which usually caused by incomplete drainage, or a less amount of pleural effusion without drainage, which means delays or improper treatment of tuberculous pleurisyis is the main cause of tuberculous empyema in clinic.

America Thoracic Association divided the empyema staging criteria for 3 period, ${ }^{2)}$ stage I for exudative phase, stage II for cellulose stage and stage III for the organizing phase. This staging method is also applicable for tuberculous empyema. Chronic tuberculous empyema belong to the stage III. Unlike non tuberculous empyema pleural disease, because of the long course of disease, chronic tuberculous empyema has thickening pleural thickening, obvious fibrosis covered with granulation tissue and caseous necrosis, cavity empyema, the visceral pleura and lung covered with organized fibreboard. The diaphragm fixed due to thickened fibreboard, restriction of breathing and chest collapse due to fiber plate pulling, intercostal narrowing, even the occurrence of spinal scoliosis. Patients are often complicated by pulmonary tuberculosis, tuberculosis 

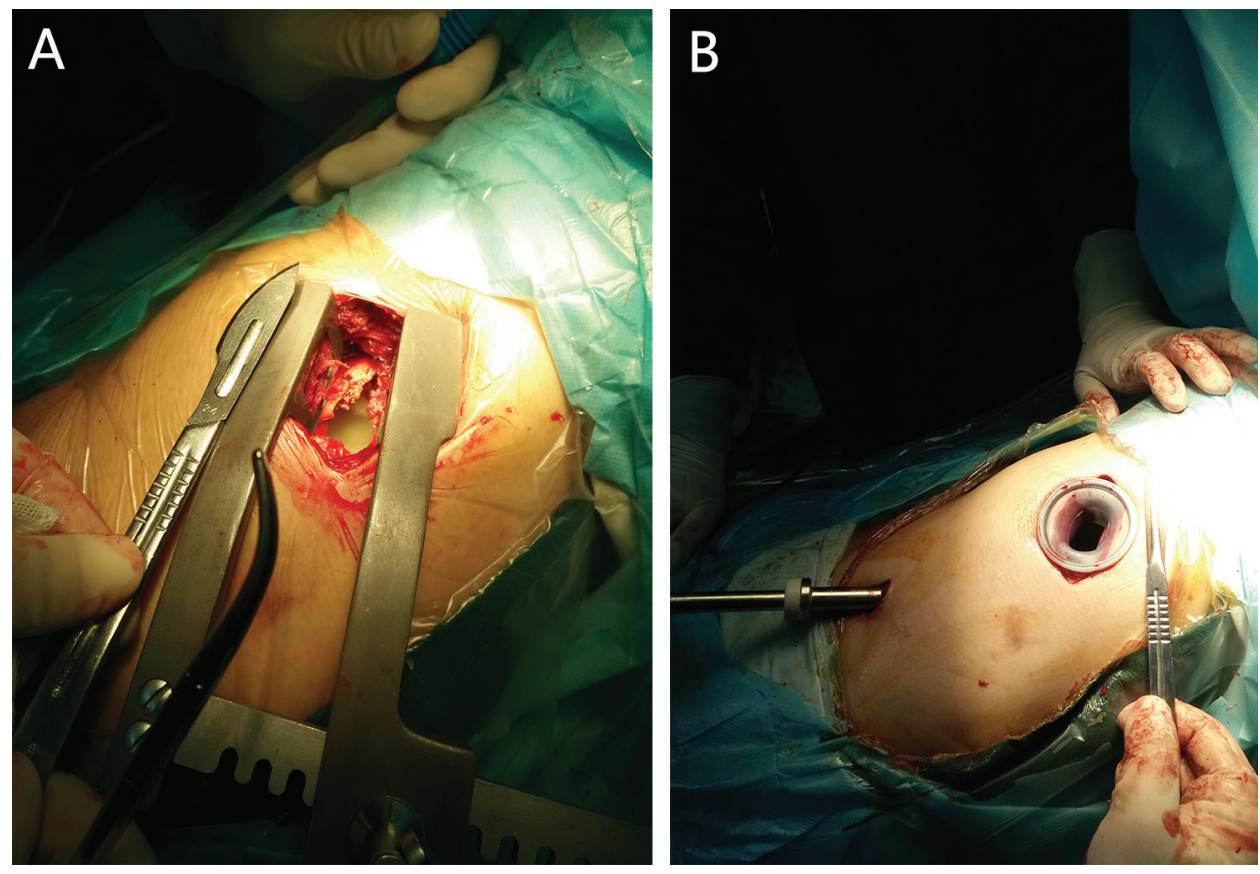

Fig. 1 Illustration of surgery. (A) Outer space of the thickening parietal pleura was exposed. (B) Small incision was used as operation hole.

of the chest wall or bone tuberculosis, which were also associated with anemia, hypoproteinemia and tuberculosis poisoning symptoms. Tuberculous empyema are usually more complex, especially when the disease developed to phase III, the curative effect of internal and invasive treatment is poor and surgical treatment is particularly important. In chronic tuberculous empyema, the fibreboard of parietal pleura is too hard for thoracoscopy to enter, so that the endoscopic operation was referred as contraindication. ${ }^{3)}$ Therefore, small incision assisted thoracoscopic hybrid surgery is considered an effective procedure for empyema thoracis. The present study aimed to explore the outcomes and complications associated with decortication by mall incision assisted thoracoscopic hybrid surgery, by retrospectively analyzing 48 patients with tuberculous empyema who underwent this procedure.

\section{Materials and Methods}

From June 2011 to January 2014, 48 cases of stage III of chronic tuberculous empyema with pleural thickening and encysted abscess cavity were chosen as experimental group. The indication for the small incision assisted thoracoscopic hybrid surgery included stage III of chronic tuberculous empyema, the hypertrophy pleural fibreboard was more than $0.5 \mathrm{~cm}$, total thoracoscope operation cannot be processed.
Among them, 28 cases were male, 20 cases were female. Ages of the patients were from 17 to 67 years old with an average of 26. Pleural fluid culture, pleural biopsy or other relevant auxiliary examination were performed before the operation to make a definite diagnosis. All cases received anti tuberculosis treatment (2-4HREZ/6-10HR) for 2 to 14 months, average of 4.7 month. If multi resistant of tuberculosis happened, sodium amimosalicylate, amikacin, fluoroquinolones, protionamide enteric-coated tablets were used for treatment.

There were no tubercular toxic symptoms, bronchopleural fistula, or active pulmonary tuberculosis before the operation. According to chest computer tomography (CT), the maximum diameter of encysted abscess cavity was from 5.0 to $15.0 \mathrm{~cm}$, with an average of $10 \mathrm{~cm}$, the thickness of the hypertrophy pleural fibreboard was from 0.5 to $2.0 \mathrm{~cm}$, with an average of $1.2 \mathrm{~cm}$. 25 cases of the same disease were chosen as control group, treated with traditional operation (empyema debridement and pleural fibreboard decortication).

In experimental group, patients were treated with intravenous anesthesia, double lumen endotracheal intubation and lateral position. According to the preoperative chest $\mathrm{CT}$, the maximum cross section of abscess cavity was determined, then incision was made around the intersection point of posterior axillary line and this plane, which 
Table 1 The operation time, amount of bleeding, the hospitalization time after operation, intubation time and the incidence of postoperative complications were compared between the two groups

\begin{tabular}{lcc}
\hline & Experimental group $(\mathrm{n}=48)$ & Control group $(\mathrm{n}=25)$ \\
\hline Operation time & $70 \pm 22 \mathrm{~min}^{*}$ & $130 \pm 32 \mathrm{~min}$ \\
Bleeding & $200 \pm 55 \mathrm{ml}^{*}$ & $400 \pm 45 \mathrm{ml}$ \\
Hospitalization time & $18 \pm 1.2$ days $^{*}$ & $28 \pm 4.5$ days \\
Intubation time & $3.5 \pm 1.5$ days* & $6.5 \pm 2.5$ days \\
Complications & 5 cases & 3 cases \\
\hline
\end{tabular}

${ }^{*} \mathrm{P}<0.05$ compared with control group.

was 4-7 $\mathrm{cm}$ through intercostal approach. When the outer space of the thickening parietal pleura was exposed, pleural outer gap was freed for $5 \mathrm{~cm}$ wide with fingers or bone stripping blunt (Fig. 1A). A small spreader was used for intercostal distraction and thickening pleura was exposed. Pleura was opened, pus and necrotic tissue in the abscess cavity were cleaned. The thick parietal pleura fibreboard around the incision, especially the thickened parietal pleura fiber board around endoscopic holes were cleaned as far as possible. Irrigated the abscess cavity, thoracoscopes was placed in a layer lower than abscess cavity. This small incision was used as operation hole (Fig. 1B). Incision retractor fixator was used to replace the spreader. Thickening fibre board of visceral pleura was blunt or sharp stripping under guide of thoracoscopes. Free adhesion of lobectomy, recruitment atelectatic lobe, mending leakage, hemostasis, drainage, closed chest. Specimen and pus were sent to examination. In control group, routine operation (Empyema debridement and fiber board decortication) was performed. Patients in both groups received antibiotics on the first three days post operation. Routine antituberculosis was used for 6-12 months after operative. Chest CT or X ray was performed in 2-3 days and 1, 3, 6, 12 months after operation. The amount of bleeding, the hospitalization time after operation, intubation time and the incidence of postoperative complications were compared between the two groups. All data were analyzed with SPSS 12.0. Data were shown with the mean \pm standard deviation $(\mathrm{x} \pm \mathrm{s})$, operation time, intubation time, the amount of bleeding, hospitalization time and other measurement were compared by student $t$-test. The incidence of postoperative complications was compared by ANOVA. $P$ $<0.05$ was considered as a statistically significant difference.

\section{Results}

In experimental group, operation time was from $45 \mathrm{~min}$ to $180 \mathrm{~min}$ with average of $70 \pm 22 \mathrm{~min}$. The amount of bleeding in operation was $200 \pm 55 \mathrm{ml}$. There was no blood transfusion or routine thoracotomy conversion in these 48 cases. Average of hospitalization time was $18 \pm 1.2$ days, intubation time was 2 to 14 days with average of $3.5 \pm 1.5$ days. There were five cases with complications after operation, with incidence of $10.42 \%$. There were three cases of pneumothorax, which were cured with continuous pleural negative pressure suction and the other two cases were pleural hematoma, which were cured with urokinase injection. In control group, operation time was from 110 to $260 \mathrm{~min}$ with average of $130 \pm 32 \mathrm{~min}$. The amount of bleeding in operation was $400 \pm 45 \mathrm{ml}$. Average of hospitalization time was $28 \pm 4.5$ days, intubation time was 3-24 days with average of $6.5 \pm 2.5$ days. There were three cases with complications after operation, with incidence of $12 \%$. There were three cases of pneumothorax, two cases were cured with continuous pleural negative pressure suction and one case was diagnosed as bronchopleural fistula and received pleural fistula repair operation. In Statistical analysis, operation time, bleeding postoperative, intubation time, hospitalization time were statistically significant different ( $\mathrm{P}<0.05)$, but the postoperative complication rate had no difference $(\mathrm{P}>0.05)$ (Table 1). Pathological examination of both groups suggested tuberculosis (Fig. 2). X ray or CT was performed at the time of discharge to confirm that the abscess cavity disappeared and recruitment of pulmonary lobe. Chest CT or X ray was performed in 1,3, 6,12 months after operation, which affirmed the curative effect (Fig. 3).

\section{Discussion}

Decortication is the ideal surgical treatment of chronic empyema, the value of video assisted thoracic surgery (VATS) operation in the treatment of early and mid-term of empyema has been very positive. ${ }^{4)}$ However, chronic empyema, especially chronic tuberculous empyema, has long course of disease, thick and adhere pleural fibreboard. The fibreboard of parietal pleural is too hard for the thoracoscope to enter. Therefore the thoracoscope for tuberculous 


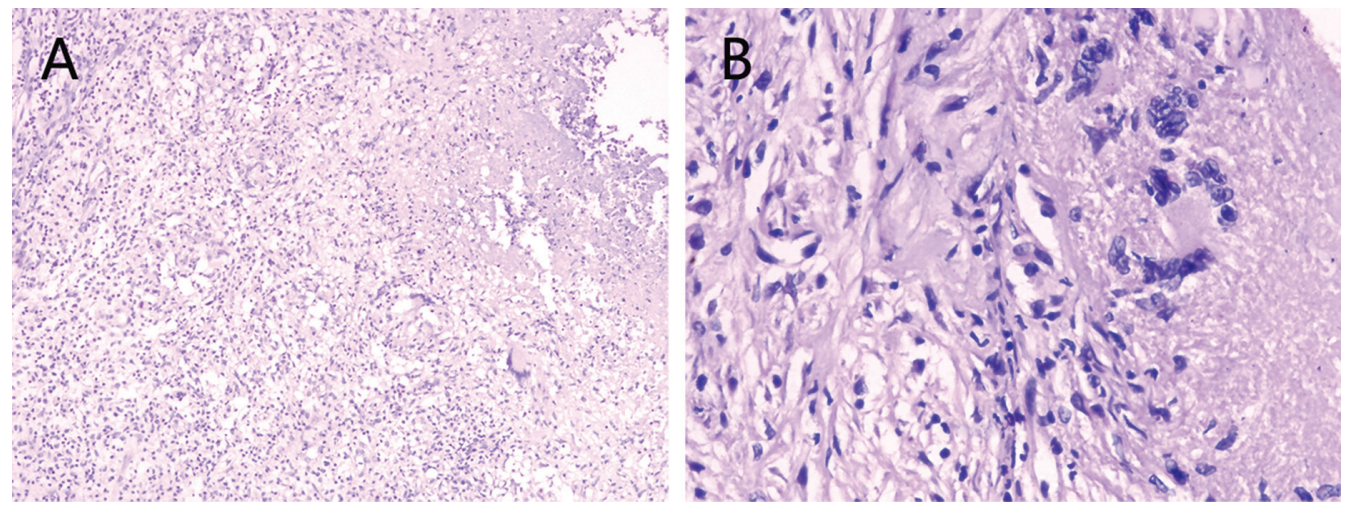

Fig. 2 HE staining for pleural tissue. (A) There were inflammatory cells infiltration $(\times 100)$. (B) There were epithelial like cells, langhans cells and lymphocytes. Exudation, necrosis and hyperplasia can be seen under the microscope $(\times 400)$.

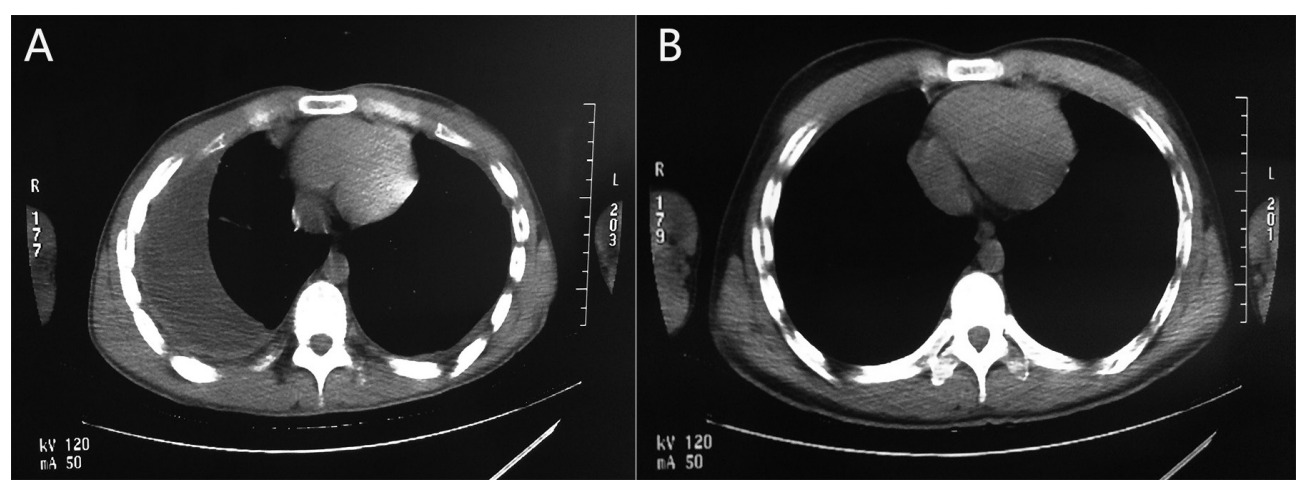

Fig. 3 CT scan for the patient with tuberculous empyema. (A) CT for the patient with tuberculous empyema before operation. (B) CT for the patient after small incision assisted thoracoscopic hybrid surgery for 12 months. CT: computer tomography

empyema was considered as contraindication. ${ }^{5)} \mathrm{Yu}$ et al. ${ }^{6}$ chose from 2003 to 2013, 183 cases of chronic tuberculous empyema patients underwent surgical treatment from Beijing Thoracic Hospital affiliated to Capital Medical University as the research object. Different chemotherapy regimens in the treatment of chronic tuberculous empyema were analyzed, 89 cases with pleural fibreboard decortication, 17 cases with thoracic close drainage plus pleural fibreboard decortication, 47 cases with thoracoplasty, 24 cases with thoracoplasty plus thoracic close drainage and six cases with pleuropulmonary resection. All cases received traditional operation treatment, endoscope minimally invasive operation intervention was not performed. The reason should be pleural fibreboard hypertrophy in chronic tuberculous empyema patients, endoscope minimally invasive operation was too difficult to perform. However, with the development of the thoracoscopic surgery, in recent years, the domestic and foreign scholars have tried to break the taboo, VATS was used for the treatment of stage III of chronic empyema.

Other surgeon in China has reported VATS was performed on stage III chronic empyema patients. As course of disease was more than 6 months long, thickness of the fibreboard was more than $1 \mathrm{~cm}$, so that the endoscopy could not enter. Then observational hole was used as the center, separate 6-8 cm circular region outside the thickened parietal pleura. Second operation hole was chosen above the abscess cavity, from outside part of parietal pleura separate another area, communicated with the observational hole region. The third operating hole was put outside the parietal pleura, after resection of thick parietal fibreboard, VATS was performed. However, this method is too complicated and it was difficult to strip parietal layer fiber board and hemostasia. Jin et al. ${ }^{7}$ has performed VATS on 82 cases of tuberculous empyema patients of stage I and stage II from 
January 2004 to June 2011. VATS was considered as safe and effective, but to the patient with more than 6 months course of disease, whose parietal fibreboard thickness was more than $1 \mathrm{~cm}$, small incision associated thoracoscopic surgery should be used, but the incision was about $12 \mathrm{~cm}$ long, trauma was relatively large and did not achieve the purpose of minimally invasive. There were also other application of VATS on chronic tuberculous empyema, ${ }^{8-11)}$ but only to stage I, stage II and the stage III with $0.5 \mathrm{~cm}$ thick fiber board. For the serves organize and more than $1 \mathrm{~cm}$ thick fiber board cases, VATS was considered as contraindication, traditional pleural decortication was the main modus operandi.

All 48 cases in experimental group, chest CT showed parietal pleural fibreboard forming, with average thickness of $1.2 \mathrm{~cm}$, the abscess cavity was enwrap. The total VATS was difficult to perform, however, small incision assisted thoracoscopy solved the operation approach problem, made the minimally invasive operation to be possible. Previous modified decortication suggested remove pus moss, necrotic tissue of parietal pleura fiber board surface, the parietal fiber board should be kept, and used to fill the abscess cavity and reduce hemorrhage from intercostal artery caused by decortication. Most of our patients were young, with average age of 26, hypertrophic fibreboard caused chest collapsed deformity, resection of parietal pleura fiber board could not only help thoracoscopy to enter, but also keep the normal thoracic morphology, restore confidence. Our results showed that this small incision assisted thoracoscopic hybrid surgery could mostly reduce the operation wound for the patients and shortened operation time, bleeding postoperative, intubation time and hospitalization time. Following points are quite important for this kind of operation: (1) The VATS for pleura decortication is difficult, so the conversion to thoracotomy should be considered. Incision should not be too low, but in the middle of abscess cavity; (2) VATS should be different as thoracotomy, total excision of hypertrophic pleura is not needed, when visceral pleura could be partly retained. Residual cavity should be eliminated as possible after pulmonary lobe was reexpanded. Apical pleural and mediastinal surface adhesion, cannot be handled if it does not affect of lung recruitment, but the diaphragmatic surface thickening fibreboard, which affected diaphragmatic activity, should be separated; (3) Operation should be carried out accurately at the anatomical level, the decortication of parietal fibreboard should be performed outside parietal pleura. The decortication of visceral fibreboard should be performed between visceral fiber board and visceral pleura;
(4) Hemostasis should be carried out at the same time with decortication of visceral fibreboard, oppression should be enough. The blood supply for parietal pleura and chest wall from the intercostal artery is rich, which caused most postoperative hemorrhage. Electrocoagulation, transfixion or hot saline gauze were used for hemostasis. (5) If serve adhesion, fibreboard hypertrophy, serve hemorrhage or serve pulmonary injury happened during minimal operation, thoracotomy should be performed immediately to guarantee the safety of the patient and reduce the complications. ${ }^{12)}$ Following complication should be paid attention: (1) Air leak, most common complications, vacuum suction could be used and pressure should be kept between -10 to $-40 \mathrm{~cm}$ water column. (2) Hemostasis. Postoperative drainage is larger than $200 \mathrm{ml} / \mathrm{h}$, more than $3 \mathrm{~h}$, no clotting blood, transfusion therapy is poor, thoracotomy should be considered. For post operation encapsulated hematoma cases, urokinase injection should be used. (3) Pulmonary atelectasis. Atelectasis without bronchopleural fistula could be solved through inhalation, expectorant, fiberoptic suction. (4) Bronchopleural fistula. Closed drainage, nutrition support, anti tuberculosis, anti infection treatment or selective operation should be performed. (5) Anti tuberculosis treatment. According to the drug sensitive result, anti tuberculosis should be kept for 6 to 12 months, quadruple medication or even more medical drugs treatment is not less than 4 months. Multi drug resistant or full resistant patients may be considered further mycobacterium species identification. Anti tuberculosis effects is always poor for Atypical mycobacteria.

In conclusion, exploration of minimally invasive treatment on stage III chronic tuberculous empyema, especially, when thickness of fibrous plate was more than $1 \mathrm{~cm}$, severe adhesion, hard parietal fibreboard were the key of VATS. We have used small incision assisted thoracotomy to make the minimally invasive hybrid operation for stage III chronic tuberculous empyema to be possible. The advantages of this method include minimal invasive, good exposure, convenient, and less bleeding. The hybrid operation method by double vision, open vision and microscope, could complete decorticate the parietal and visceral pleura, promote the pulmonary recruitment. Compared with traditional decortication thoracotomy, operation time, amount of bleeding, postoperative intubation time and hospitalization time were significantly reduced. Moreover, our incision was smaller, retain the rib, without transfusion and has the same incidence of complications as traditional operation, which means post-operation complications was associated with pulmonary quality, the degree of pleural thickening 
and adhesion and the surgeon's technology. Besides, two cases of experimental group had parcel hematoma, meanwhile, it did not happened in control group. The reason should be hemostasis in VATS was more difficult than traditional operation, due to the short view under thoracoscopy and this was also the common failing for all VATS operation.

Therefore, empyema staging is not so important for VATS operation. For stage I and II, VATS has obvious advantage, but for stage III, small incision assisted thoracoscopy could also reach the medical purpose. Whether it is total VATS, small incision assisted surgery or traditional surgery, the purpose of operation is to eliminate the abscess cavity and promote pulmonary recruitment.

\section{Conflict of Interest}

None.

\section{References}

1) Raymond D. Surgical intervention for thoracic infections. Surg Clin North Am 2014; 94: 1283-303.

2) M N, Sharathchandra BJ, Thrishuli PB. ICD Versus VATS as Primary Treatment in Fibrinopurulent Stage of Empyema Thoracis. J Clin Diagn Res 2013; 7: 2855-8.

3) Chung JH, Lee SH, Kim KT, et al. Optimal timing of thoracoscopic drainage and decortication for empyema. Ann Thorac Surg 2014; 97: 224-9.
4) Kaiser D, Ennker IC, Hartz C. Video-assisted thoracoscopic surgery-indications, results, complications, and contraindications. Thorac Cardiovasc Surg 1993; 41: 330-4.

5) Yamaguchi M, Takeo S, Suemitsu R, et al. Video-assisted thoracic surgery for fibropurulent thoracic empyema: a bridge to open thoracic surgery. Ann Thorac Cardiovasc Surg 2009; 15: 368-72.

6) Yu D, Han Y, Liu Z. Clinical effect on chronic tuberculosis empyema in treatment of surgical operation. Zhonghua Yi Xue Za Zhi 2014; 94: 2441-3.

7) Lu J, Jin MH. Minimally invasive chest incision treatment of tuberculous empyema. J Clin Pulm Med 2005; 10: 610-1. (in Chinese)

8) Petrakis IE, Kogerakis NE, Drositis IE, et al. Videoassisted thoracoscopic surgery for thoracic empyema: primarily, or after fibrinolytic therapy failure? Am J Surg 2004; 187: 471-4.

9) Lardinois D, Gock M, Pezzetta E, et al. Delayed referral and gram-negative organisms increase the conversion thoracotomy rate in patients undergoing video-assisted thoracoscopic surgery for empyema. Ann Thorac Surg 2005; 79: 1851-6.

10) Wurnig PN, Wittmer V, Pridun NS, et al. Video-assisted thoracic surgery for pleural empyema. Ann Thorac Surg 2006; 81: 309-13.

11) Barbetakis N, Paliouras D, Asteriou C, et al. eComment: the role of video-assisted thoracoscopic surgery in the management of tuberculous empyemas. Interact Cardiovasc Thorac Surg 2009; 8: 337-8.

12) Farjah F, Symons RG, Krishnadasan B, et al. Management of pleural space infections: a population-based analysis. J Thorac Cardiovasc Surg 2007; 133: 346-51. 